\title{
VENTAJAS E INCONVENIENTES DE LA FORMACIÓN ONLINE
}

\section{ADVANTAGES AND DISADVANTAGES OF ONLINE TRAINING}

\author{
Verónica Marín Díaz*; Eloísa Reche Urbano** y \\ Guadalupe A. Maldonado Berea \\ Universidad de Córdoba y Universidad Veracruzana
}

\section{RESUMEN}

Los cambios tecnológicos por los que la sociedad, en general, y la educación, en particular, han estado atravesando implican la renovación de los sistemas de enseñanza en todos sus niveles. La aparición-renovación de un sistema tradicional de enseñanza como la educación a distancia cobra cada día mayor importancia si prestamos atención a todos los aspectos que implican el desarrollo de aprendizajes. En las líneas que a continuación presentamos hacemos una exposición de motivos por los que creemos que la formación online, el aprendizaje en red o el e-learning han de ser vistos como una herramienta que brinda una nueva forma de ver, sentir y entender la enseñanza universitaria.

Palabras clave: e-learning, proceso de enseñanza-aprendizaje, comunidades de aprendizaje, profesor universitario.

\begin{abstract}
The technological changes that society, in general, and,education in particular, have been experiencing demand that all levels of the education system be renovated. The emergencerenovation of a traditional education system such as distance education has become increasingly important each day if we take into account all the aspects involved in how people learn. elow we explain why we believe that online training, learning through networks or e-learning should be considered as a tool that provides a new way of seeing, feeling and understanding university education.
\end{abstract}

Keywords: e-learning, learning-teaching process, learning communities, university teacher. 


\section{INTRODUCCIÓN}

La sociedad de la información y la comunicación que hoy poco a poco vamos construyendo y reconstruyendo, ha sido fruto de diversas revoluciones que hace más de una década Cabero (1996) y Vergés (1998) señalaban. Todas ellas presentaban, a nuestro juicio, un aspecto común y peculiar que no es otro que la determinación, en cierta manera, de los avances tecnológicos que nos han hecho llegar hasta el momento actual. Esta nueva sociedad demanda formas de organizar la vida social, política, económica y educativa de los países y, en consecuencia, nuevos profesionales con una amplia gama de competencias, entre ellas, la denominada competencia digital.

Centrando nuestra atención en el ámbito educativo, la incorporación de la tecnología educativa y la formación de la competencia tecnológica hunde sus raíces en el no muy lejano siglo XX. Si en torno a la década de los 50 la educación se convirtió en un elemento cardinal de las políticas desarrolladas por los gobiernos, hoy las circunstancias no han cambiado sustancialmente pues continúa siendo un eje principal; sin embargo, la diferencia estriba ahora en el amplio abanico de recursos que propician su desarrollo. Esta evolución nos ha hecho hablar de términos como Nuevas Tecnologías (NNTT), Tecnologías de la Información y la Comunicación (TIC) y de Tecnología Educativa (TE). Todas ellas abarcan la red, Internet, Web 2.0, e-learning, plataformas de teleformación, entornos personales de aprendizaje (PLE), comunidades virtuales de aprendizaje, wikis, blogs, etc.

En la actualidad las TIC se contemplan como una herramienta que, dado su carácter transversal, propician un alto nivel de aprendizaje (Pires y Moreira, 2012) tal y como refleja la investigación llevada a cabo por Ahmadi, Keshavauzi y Forouten en 2011.

*vmarin@uco.es

**e.reche@magisteriosc.es

[RIDU]: Revista Digital de Investigación en Docencia Universitaria Dic. 2013 - Año 7 - Nro. 1 | LIMA (PERÚ)
Por otro lado, la incorporación de las TIC y de la red Internet a los sistemas educativos permite que el tan conocido binomio espaciotiempo genere una adaptación de los procesos de aprendizaje de los individuos, además de ser entendidas y sentidas como un elemento que facilita el acceso al conocimiento, a su creación y difusión. Como sostiene Palomares et al. (2007, 71) las tecnologías de la información y la comunicación en estos momentos "favorecen la asociación de ideas y la creatividad, el desarrollo de enfoques de aprendizaje profundo, posibilitando operaciones lógicas de análisis, síntesis, abstracción, inducción y deducción, lo que da lugar a un desarrollo integrado del pensamiento".

La incorporación de Internet a la dinámica educativa supone el aprendizaje de estrategias que ayuden en la búsqueda y tratamiento de la información, así como a la difusión de nuevo conocimiento que de ella se genera. Es decir, hablamos de competencia informacional. Compartimos con Ahmadi, Keshavauzi y Forouten (2011) la idea de que la red Internet se caracteriza también por facilitar el establecimiento de interacciones sociales internacionales, y por apoyar y dar a conocer descubrimientos.

También hacen referencia, de forma explícita, al diseño de las metodologías, contenidos, organización escolar, cultura y los ámbitos de decisión del sistema educativo, aspectos que se reflejan en las variables, aportadas por Cabero $(2008,24$,) y que inciden en los procesos de formación a través de la red. Es decir, dentro de estos nuevos entornos, hablamos de: "sentido de comunidad/ sociabilidad/intervención social; contenidos; metodología/diseño/estrategias didácticas; aspectos comunicativos/herramientas de 
comunicación; entorno tecnológico; modelo de evaluación; soporte institucional/aspectos organizativos; competencias tecnológicas; centrado en el estudiante/activo/colaborativo/ participativo; e-actividad; papel del profesor/ tutor; papel del estudiante". Todos estos elementos harán que el desarrollo de los procesos de formación en los que intervengan las TIC se caractericen por su accesibilidad, flexibilidad, apertura, multiplicidad de vías de comunicación, entre otros.

Pero no olvidemos el aspecto sobre el que, a nuestro entender, pivotan las tres variables básicas de la formación con TIC, que no es otro que la motivación, modulado este por quién aprende, el tipo de material didáctico empleado y la propia acción del docente. Todo ello está enmarcado desde dos visiones, una que implica un seguimiento del desarrollo del proceso de aprendizaje y otra que proporciona oportunidades de participar en los procesos educativos desde su momento inicial (Demirbolat, 2006).

\section{De la educación online}

La aparición de un nuevo sistema de formación, que cubra aquellos aspectos que la tradicional educación a distancia albergaba, ha supuesto una revolución en los formatos de transmisión del conocimiento, así como en el desarrollo de procesos de enseñanza-aprendizaje de carácter sincrónico y asincrónico.

El desarrollo de la red de redes, Internet, como elemento conductor de todo lo anteriormente señalado, ha provocado una conmoción, que si bien en épocas pretéritas no se imaginaba sus dimensiones, -aún habiendo pasado por diferentes fases o revoluciones-. Hoy el punto álgido de este tipo de formación tiene su epicentro en torno al año 2000, momento en que la Comisión Europea pone el acento sobre la importancia social, además de pedagógica, de las TIC (Gavari, 2006), defendiendo a través de diferentes programas la integración de las mismas en el sistema educativo y formativo europeo.

Todas estas iniciativas se ponen en marcha a través de diversas líneas de acción, -"fomento de la alfabetización digital; los campus virtuales europeos; el hermanamiento electrónico de centros de enseñanza europeos y el fomento de la formación del profesorado; y las acciones transversales para la promoción del e-learning en Europa" (Gavari, 2006, 190)-, que proporcionan una relación complementaria (educación-e/m/b-learning) dado que la primera aporta los modelos pedagógicos a las segundas, y estas, a aquella, la creatividad, el dinamismo y la renovación continua del saber.

Las innovaciones que estas propuestas formativas han desarrollado o puesto en marcha en los últimos tiempos han ido dirigidas principalmente hacia:

a) La transformacióny velocidad de cambio de los contenidos, principalmente en su diseño. b) Instancias educativas reglamentadas dejan de ser las únicas proveedoras de la formación, apareciendo otros como los medios de comunicación de masas, -tradicionales/convencionales o novedosos-, y las instituciones empresariales, -sindicales o corporativas-.

c) La creación de entornos de formación altamentetecnificados,ypuestosadisposición tanto del profesorado como para el alumnado. d) Los cambios en las concepciones del aprendizaje, dado que ahora se habla de una formación centrada en el estudiante.

e) La articulación del aprendizaje en torno a la concepción de la comunicación sincrónica y asincrónica. 
f) Formación del estudiante en nuevas competencias y capacidades digitales e informacionales.

g) Necesidad de alfabetizaciones diferentes a las actuales, -alfabetizaciones digitales-.

h) la transformación del rol del profesor.

i) Los cambios en las estructuras organizativas.

j) La necesidad de configurar redes de formación.

k) Entornos de formación cada vez con mayor grado de iconicidad, como por ejemplo, los entornos en 3D tipo SecondLife.

l) La articulación del aprendizaje, combinando tanto lo analógico y lo virtual como lo presencial y a distancia.

Autores como Hernández, Acosta, Rodríguez, González y Borges ya en 2003 pensaban que la teleformación podía ser entendida como "el uso de herramientas telemáticas en la enseñanza, la cual puede combinar sesiones presenciales como sesiones no presenciales e incluir distintos aspectos instruccionales (conceptualización teórica, práctica, contactos sincrónicos y asincrónicos, etc.)” (1). En esta línea, la educación online permite ampliar las perspectivas de aprendizaje, que van más allá de los tradicionales paradigmas de formación. Supone asumir que las dos funciones principales serán la flexibilización de los procesos comunicativos y el trasvase continuo de información.

En consecuencia, los procesos de aprendizaje derivados de la formación a distancia irán más allá de una simple recepción de píldoras de contenido. Esta debe ir más lejos, ha de potenciar un aprendizaje más exigente en los resultados, todo ello producto de una guiada planificación por parte del docente, para que el estudiante pueda aprender haciendo y desarrolle estrategias de trabajo cooperativo, superando, asimismo,

*vmarin@uco.es

**e.reche@magisteriosc.es

[RIDU]: Revista Digital de Investigación en Docencia Universitaria Dic. 2013 - Año 7 - Nro. 1 | LIMA (PERÚ) la concepción colaborativa de este, para que adquiera destrezas que favorezcan su autonomía formativa.

Este sistema formativo se caracteriza, principalmente, por el continuo proceso de construcción y reconstrucción del aprendizaje, así como del conocimiento que realiza el estudiante y el profesorado; todo ello implica la desaparición de terminologías (educación a distancia) y la aparición de otras (e-learning, b-learning,m-learning, ...), cambio de roles tanto en el docente como en el estudiante (Valtonen et al., 2011), creación de espacios comunes de trabajo (Salinas, 2004) y aprendizaje flexible, independiente y/o colaborativo-cooperativo.

Con respecto al último, se están produciendo transformaciones en la propia idea que manejamos sobre este; frente a concepciones tradicionales de asociación e interpretación, del aprendizaje con la mera incorporación memorística de la información, o la simple modificación de la conducta y habilidades en los estudiantes. Encontramos que, en la actualidad, las concepciones que empleamos sobre el aprendizaje tratan de asumir principios y acciones, tales como que:

- El conocimiento no es incorporado mecánicamente a la estructura cognitiva del sujeto, sino que se construye a partir de nuestras interacciones con el medio ambiente y el entorno sociocultural en el cual estamos inmersos.

- El aprendizaje es, por una parte, un proceso activo, donde la participación del estudiante es un elemento clave para alcanzarlo, y por otra, un proceso social donde el sujeto en interacción con sus compañeros y con el resto de variables modifica su estructura cognitiva.

- El conocimiento no es independiente del ambiente y contexto en el cual se produce. No es aislado sino contextual, y 
el mismo contexto se convierte en parte del mismo.

- Los contextos de formación deben ser auténticos y significativos para el aprendizaje, y no simplemente depositarios de información. La experiencia que se desarrolla en la acción educativa, es percibida como central, y los contenidos que se llegan a transmitir como periféricos.

- El conocimiento se adquiere a partir de acciones de interacción y no del aislamiento. Esta interacción debemos percibirla desde una perspectiva amplia: con personas, con tecnologías, con objetos, etc.

- El conocimiento viene matizado por diferentes elementos entre los que se encuentran la cultura, la comunidad en la que vivimos y las herramientas (tecnológicas y sociales) que manejamos y que producimos de forma sistémica.

- De un conocimiento centrado en expertos, se ha pasado a un conocimiento distribuido, donde el experto pasa a ser un mediador, un guía.

- Del aprendizaje individual al aprendizaje colectivo y/o colaborativo, por medio del mismo se desarrollarán elementos tan cruciales como las relaciones multidisciplinares.

- Asunción de que si hay hoy una teoría educativa que explique la adquisición del conocimiento, posiblemente sea su metateoría.

Asumiendo algunos de los principios que hemos formulado, la enseñanza online del futuro debe tener en cuenta y potenciar una formación activa, constructiva, colaborativa, intencional, contextualizada y reflexiva.

Si es cierto que para que todo esto se produzca (desarrollo de conocimiento y de procesos efectivos de aprendizaje) será necesario que, además de realizarse en red, las alternativas propuestas vayan más allá del paradigma o paradigmas formativos de corte tradicionalista y pongan especial énfasis en la búsqueda y construcción de la universidad del futuro. En esta línea, Prattini y Mengual $(2008,17)$ señalan las innovaciones que este tipo de formación ha ido presentando desde que en el año 2000 se planteó como una nueva estrategia formativa puesta en marcha por las universidades. Entre los aspectos que las caracterizan destacan los siguientes:

- Genera nuevo conocimiento.

- Es propiedad del estudiante.

- Crea comunidades de aprendizaje.

- Es el resultado y una herramienta para soportar una 'sociedad' (partnership).

- Se basa en el contexto del estudiante y sus logros previos.

- Estimula la creatividad del estudiante incrementando la dimensión espontánea y lúdica del aprendizaje.

- Enriquece el papel de los profesores y las facilidades del aprendizaje.

- Se centra en la calidad y contextos de aprendizaje.

- Está embebido en los procesos organizacionales y sociales de transformación.

- Llega y motiva a aquellos que no están aprendiendo.

El aprendizaje que se deriva de este tipo de procesos se caracteriza, según Wangy Chin (2011), por promover el intercambio y el enriquecimiento de este. Aspecto que, por otra parte, obligaráa tener en cuenta los componentes técnicos, curriculares, organizativos, institucionales, didácticos, etc., permitiendo así, una mayor adaptación de los tradicionales modos de enseñanza a estas nuevas perspectivas, lo que, al mismo tiempo, dará nuevos roles a los docentes y a estudiantes. 


\section{Ventajas e inconvenientes de la formación online}

La formación en red (e/b/m/p-learning) debe, a nuestro juicio, ser entendida como un ambiente cooperativo de aprendizaje, en el cual se potencie la adquisición y desarrollo de competencias, en general, y la de corte tecnológico, en particular. La investigación realizada por Koory en 2003 pone de relieve que muchos estudiantes mejoran sus resultados de aprendizaje a través de la formación online, es por ello que algunos autores consideren que la educación a distancia nace para superar diferentes brechas, bien sean sociales, económicas, geográficas, etc. (Pino, 2008).

Pero todo recurso o metodología presenta aspectos negativos o inconvenientes así como aspectos positivos o ventajas. A través de la revisión de la literatura realizada (Muñoz, 2004; Cebrián, 2004; Cabero, 2005, 2006; Pryer y Bitter, 2008; Revuelta y Pérez, 2009) podemos encontrar los siguientes puntos de interés en ambos aspectos (Ver tabla 1).

Como podemos observar, los beneficios que conlleva esta modalidad de formación son ampliamente significativos. En los últimos años se ha puesto especial énfasis en facilitar escenarios de aprendizaje que favorezcan, por un lado, la simultaneidad de actividades teniendo presente la posibilidad de compatibilizarlas con el ámbito laboral, y en segundo lugar, proporcionar el acceso a un amplio abanico de ofertas formativas, independientemente del lugar de residencia.

Por su parte, en el proceso de enseñanzaaprendizaje es ineludible el seguimiento del trabajo del alumnado y la formación online facilita su tutela de manera grupal e individual, dando lugar a la retroalimentación a través de las diversas herramientas que propician la interacción entre profesorado y estudiantes, así como entre estos últimos, bajo la supervisión del docente.
Por otro lado, una de las claves para el funcionamiento eficaz se sustenta en la actualización del contenido y la necesidad de un continuo mantenimiento del sistema con la consabida inseguridad que la red conlleva, lo que derivan en el esfuerzo y en la sobrecarga de trabajo del profesorado.

Indudablemente el e/b/m-learning proveerá de oportunidades formativas (Boom Rusman, Van der Klink y Tattersall 2005), pero debemos ser conscientes de que, para el desarrollo de un sistema de aprendizaje basado en red, se han de superar otros inconvenientes, además de los señalados anteriormente, tales como el coste, que inicialmente suele ser elevado; un mayor empleo de la tutoría; la comunicación se convierte en impersonal dejando a la imaginación la comunicación no verbal; la falta de hábito para trabajar en red, así como el desconocimiento de los recursos tecnológicos y de su uso, la baja calidad de algunas ofertas formativas basadas en este sistema, así como la escasa formación de los docentes en este sistema de trabajo (Hernández et al, 2003; Cebrián, 2004; Cabero, 2006; Gallego y Alonso, 2007).

De todos los aspectos antes señalados, la flexibilidad es, quizás, la característica definitoria de este tipo de formación. Gallego y Alonso $(2007,215)$ puntualizan esta flexibilidad en tiempo, contenido, requisitos de admisión, instituciones y recursos e impartición y logística, los cuales concretamos en la tabla 2.

El desarrollo de la formación en red implica el trabajo en equipo, donde se compartirán y se elaborarán informaciones, además de tomar decisiones relevantes para el correcto aprendizaje, teniendo como fin principal resolver las desventajas que genera el sistema y que anteriormente señalábamos. 
Tabla 1

Ventajas e Inconvenientes de la formación en red

\begin{tabular}{|c|}
\hline Ventajas \\
\hline Elimina distancias físicas \\
\hline Flexibilidad horaria \\
\hline Favorece la interacción \\
\hline Acceso instantáneo e ilimitado a recursos \\
\hline Interactividad \\
\hline Control de la comunicación \\
\hline Potenciadora del trabajo cooperativo \\
\hline Flexibilidad \\
\hline Posibilidad de adaptación del proceso de aprendizaje \\
\hline Personalización del proceso de aprendizaje \\
\hline Resolución inmediata de problemas \\
\hline Ejercicio continuado de reflexión \\
\hline Satisfacción \\
\hline Construcción de nuevos conocimientos de forma inmediata \\
\hline Promueve múltiples perspectivas sobre el empleo de la información obtenida \\
\hline Facilita la interacción entre diferentes áreas de conocimiento \\
\hline Facilita el uso y consumo de materiales \\
\hline Desfocalización del conocimiento \\
\hline Diferentes formas de comunicación (sincrónica y asincrónica) \\
\hline Registro continuado del progreso formativo \\
\hline
\end{tabular}

Comunicación impersonal

Dificultad en la resolución de problemas

Coste inicial de mantenimiento, conexión

Complejidad en las condiciones de privacidad

Escasa cooperación por parte de los docentes

Ausencia de contacto directo

Falta de motivación

Barreras psicológicas (resistencia al cambio)

Falta de formación por parte de los usuarios

Escasa calidad de los cursos y contenidos

Escasa existencia de tutores virtuales

Falta de hábitos de enseñanza-aprendizaje

Mayor dedicación por parte del docente

Soledad

Disminución de la calidad de la formación

Elevada ratio profesor-alumno

Fuente: Elaboración propia. 
Tabla 2

Flexibilidad de la enseñanza en red

Flexibilidad

\begin{tabular}{ll}
\hline & -Para empezar y terminar el curso. \\
Tiempo & -Para estudiar el curso. \\
& -Ritmo de estudio. \\
& -Temas del curso. \\
& -Secuencia de las diferentes partes del curso. \\
& -Tamaño (cantidad del contenido) del curso. \\
Contenido & -Nivel del curso. \\
& -Estándares de evaluación \\
Requisitos de admisión & -Condiciones para participar en el curso. \\
& -Organización social del aprendizaje. \\
Instituciones y recursos & -Lengua que se utiliza durante el curso. \\
& -Recursos de aprendizaje. \\
\hline Impartición y logística & -Tiempo y lugar donde está disponible la ayuda. \\
& -Método para obtener ayudas y apoyo. \\
& -Tipos de apoyo disponibles. \\
& -Canales de impartición del curso. \\
\hline
\end{tabular}

Fuente: Elaboración propia.

En todo este mapa de cambios, el Reflexiones finales

docente, como ya hemos señalado antes, asume un nuevo rol que debe ser entendido como una ventaja que potenciará el desarrollo de este tipo de formación. El profesor se hace cargo de nuevos deberes comola realización de un seguimiento diario o semanal del estudiante, con el consiguiente aumento de horas dedicadas a la corrección de trabajos, potenciando una evaluación más justa, según señala Pino (2008). Y el estudiante, por su parte, será quien decida el orden secuencial de su aprendizaje y del acceso a la información. La efectividad de dicho aprendizaje vendrá determinada también por el cambio de pensamiento de ambos actores (Starkey, 2011).

El crecimiento de la sociedad de la información y del conocimiento conlleva el desarrollo de las habilidades digitales necesarias para que esta crezca en armonía. Supone un aumento de la cultura digital frente a la impresa, así como la concepción de que los procesos de enseñanzaaprendizaje se pueden realizar fuera de las fronteras espacio-temporales.

Compartimos con Tavukeu, Anap y Ozcan (2011) que el desarrollo de las TIC, y en consecuencia, de la red Internet, han posibilitado una nueva forma de comunicación con otros sujetos a los cuales se les provee de una oportunidad de aprendizaje con personas con quienes no se comparte espacio físico.

*vmarin@uco.es 
Y tal y como afirmaba Cabero en 2005, esa oportunidad de formación a distancia, que bien puede o no ser de carácter reglamentado, debe posibilitar un "aprendizaje interactivo, flexible y accesible, a cualquier receptor potencial" (26).

No debemos olvidar que el aumento de las fuentes de conocimiento hoy hacen casi imposible que la formación superior, proporcionada por los centros educativos en estos momentos, sea abarcada en su totalidad, de ahí la necesidad de buscar nuevas formas de enseñanza que, además, potencien el aprendizaje a lo largo de toda la vida, -no solo de los estudiantes también debe producirse un continuo reciclaje de los docentes-, y el desarrollo de las competencias tecnológicas necesarias para hacer frente a las demandas de este nuevo sistema.

El empleo de una formación online, -llámese e-learning, b-learning, m-learning-, implica por parte del profesorado asumir una serie de compromisos pero que, como sostiene Pino $(2008,93)$, conllevan una cadena de obligaciones y de beneficios. Esta autora señala como obligaciones diseñar objetivos y competencias en cada unidad didáctica o tema a desarrollar, de manera que el alumnado sepa lo que debe saber hacer o conseguir dominar de ese contenido; realizar un seguimiento por lo menos semanal del alumnado lo que lleva parejo el aumento considerable del tiempo de corrección; determinar claramente cuáles son las evidencias que el alumnado debe presentar.

Dichas obligaciones implican como beneficios la realización de una evaluación más acorde con la realidad formativa y precisa, así como el desarrollo de un aprendizaje que motiva más al alumno y que está en consonancia con las demandas sociales y laborales del momento.

Por otro lado, la flexibilidad que conlleva esta modalidad de enseñanza brinda la

*vmarin@uco.es

**e.reche@magisteriosc.es

[RIDU]: Revista Digital de Investigación en Docencia Universitaria Dic. 2013 - Año 7 - Nro. 1 | LIMA (PERÚ) oportunidad de formación a un amplio número de estudiantes con especificidades cada vez más diversas, entre ellas, las que se derivan de las carencias en este tipo de experiencias formativas. Se mantienen las características iniciales de la formación online, pero se añaden nuevos criterios, como la adquisición y desarrollo de la competencia digital y la competencia informacional.

La conjugación de las TIC y el conocimiento didáctico del contenido podrán ser entendidas como los ingredientes necesarios para poder generar una formación acorde con el momento tecnológico que estamos viviendo. En consecuencia, la calidad de los resultados esperados del proceso de enseñanza-aprendizaje podrán ser mejorados (Bartolomé, 2004), por lo que se debe abogar por la búsqueda de las condiciones necesarias para que estas mejoras se produzcan.

Debemos superar la idea de concebir esta formación como una herramienta exclusiva para que aquellas personas que no pueden cursar una formación reglada presencial lo hagan. Desde estas líneas afirmamos que los sistemas de formación apoyados en plataformas online van más allá de proporcionar un listado de contenidos, implican ayudar a desarrollar competencias tales como el trabajo en equipo, el liderazgo, la búsqueda continua, etc.

En definitiva, las principales premisas que guiarán la enseñanza virtual serán la apertura y la flexibilidad (Palloff y Pratt, 2001).

\section{REFERENCIAS}

Ahmadi, S., Keshavarzi, A. \& Foroutan, M. (2011). The application of information and communication technologies (ICT) and its relationship with improvement in teaching and learning. Procedia 
Social and Behavioral Sciences, 28, 475-480.

Bartolomé, A. (2004). Blendend learning. Conceptos básicos. Pixel Bit, Revista de Medios y Educación, 23, 7-20.

Boom, J., Rusman, E., Van der Klink \& Tattersall, C. (2005). Developing a critical view on e-learning trend reports: trend watching or trend setting? International Journal of Training and Development, 59 (3), 205-211. http://www.qou. edu/arabic/researchProgram / e $\mathrm{L}$ e a $\mathrm{rn}$ i n g R e s a r c h s / developingACritical.pdf.

Cabero, J. (1996). Nuevas tecnologías, comunicación y educación. Edutec, Revista Electrónica de Tecnología Educativa, 1. http://www.uam.es/ personal_pdi/stmaria/iparedes/ lecturas/cabero.htm,

Cabero, J. (2005). Formación del profesorado universitario en estrategias metodológicas para la incorporación del aprendizaje en red en el espacio de educación superior. Memoria de investigación. http://tecnologiaedu. us/biblig.

Cabero, J. (2006). Bases pedagógicas del e-learning. Revista de Universidad y Sociedad del Conocimiento, (RUSC) 3 (1). http://www-.uoc.s/ruosc/3/1/ dt/esp./cabero.pdf.

Cabero, J. (2008). La investigación en educación a distancia en los nuevos entornos de comunicación telemáticos. International Journal of Social Escientes \& Humanities, XVIII (2), 13-34.

Cebrián, M. (2004). Impacto de las tecnologías de la información y comunicación en la Universidad. Bordón, 56 (3/4), 587600.

Demirbolat, A. (2006). Education faculty students' tendencies and beliefs about the teacher's role in education: a case study in a Turkish university. Teaching and Teacher Education, 22 (8), 10681083.

Gallego, D. \& Alonso, C. (2007). La educación a distancia en los nuevos contextos socioeducativos. En J. Cabero (coord.). Tecnología educativa. (pp. 195-217). Madrid: McGraw-Hill.

Gavari, E. (2006). Los principios rectores del espacio europeo de educación superior virtual. Revista Electrónica Teoría Educativa, 7 (2), 185-197.

Hernández, Mạ C., Acosta, M. C., Rodríguez, E., González, E., \& Borges, M. (2003). Uso de las TICS y percepción de la teleformación en el alumnado universitario: una perspectiva diferencial en función del género $\mathrm{y}$ del ciclo de la carrerera. Interactive Educational Multimedia, 7. Rescatado de http://www.ub.edu/multimedia/ iem/down/c7/Use_and_Perception_ of_ICTs_(SPA).pdf.

Koory, M.A. (2003). Differences in learning outcomes for the online and F2F version of on introduction to Shakespeare. Journal Asynchronous Learning Networker, 7 (2), 18-35.

Muñoz, L. A. (2004). Las tecnologías de la información y la comunicación y la formación en entornos virtuales. Revista Complutense de Educación, 15 (1). 51-74.

Palomares, T., Fernández, K., Modroño, J. I., González, J., Sáez, Fco. J., Chica, Y., et al. (2007). Las tecnologías de la información y comunicación en la enseñanza universitaria: influencia sobre la motivación, el autoaprendizaje y la participación activa del alumno. Revista Psicodidáctica, 12 (1), 51-78.

Piattini, V. \& Mengual, L. (2008). Universidad 
digital 2010. (5-27). En J. Lavaine \& L. Mengual. (dirs.). Libro blanco de la Universidad digital 2010. (pp. 5-27). Barcelona, Arial.

Pino, M. (2008). Aplicaciones de herramientas de e-learning a la docencia presencial. Revista de Formación e Innovación Educativa Universitaria, 1 (4). 87-95.

Pires, E. \& Moreira, F. (2012). The integration of information in communication technology in schools. Online safety. Procedia Technology, 5, 59-66.

Pryer, C. \& Bitter, G.G. (2008). Using multimedia to teach inservice teachers: impact on learning, application and retention. Computer in Human Behavior, 24 (6), 2668-2681.

Revuelta, F.I, \& Pérez, L. (2009). Interactividad en los entornos de formación online. Barcelona: UOC.

Salinas, J. (2004). Cambios metodológicos con las TIC. Estrategias educativas $\mathrm{y}$ entornos virtuales de enseñanzaaprendizaje. Bordón, 56 (3/4), 469480.

Starkey, l. (2011). evaluating learning in the $21^{\text {st }}$ century: a digital age learning matrix. Technology, pedagogy and education 20 (1), 19-39.

Valtonen, T., Hacklin, S., Dillon, P., Vesisenaho, M., Kukkonen, J. \& Hietanen, A. (2012). Perspectives on personal learning environments held by vocational students. Computers \& Education, 58 (2), 732-739.

Vergés, M (1998). La era de los programas de informática educativa. En M. Aguirregabiria (coord.). Tecnología y educación. (pp. 131-137). Madrid: Nareca.

Wang, H.C. \& Chiu, Y. F. (2011). Assessing e-learning 2.0 system success. Computes \& Education, 57, 1790-1800.
Tavukeu, T., Anap. I \& Ozcan, D. (2011). General overview on distance education concept. Procedia Social and Behavioral Sciencies, 15, 3999-4004. 